Henriette Engelhardt

\title{
Zum Wandel der Korrelation von Fertilität und Frauenerwerbstätigkeit in Raum und Zeit: Eine empirische Analyse unter Berücksichtigung der Effekte ausgewählter sozialer Indikatoren
}

\author{
On the changing correlation between fertility and female employment over \\ space and time: A pooled time-series analysis on the impact of social \\ indicators
}

\begin{abstract}
Zusammenfassung:
In den OECD-Ländern hat sich die Korrelation zwischen der Gesamtfertilitätsrate und der Frauenerwerbsquote über die Zeit von einem negativen Wert vor den 1980er Jahren zu einem positiven Wert gewendet. Dieser Wechsel ist offensichtlich durch unbeobachtete länderspezifische Heterogenität und durch Heterogenität in der Beziehung zwischen Fertilität und Erwerbstätigkeit über die Zeit bedingt. Die Determinanten dieser zeit- und länderspezifischen Heterogenität sind jedoch unklar. Basierend auf Daten aus 16 europäischen Ländern werden in der vorliegende Studie zusammengefasste Zeitreihenmodelle mit fixen Länderund Zeiteffekten geschätzt und die Veränderung des Effekts der Erwerbsbeteiligung von Frauen für Indikatoren des Arbeitsmarktes, der Bildungsbeteiligung von Frauen, proximale Determinanten der Fertilität sowie sozialpolitische Indikatoren untersucht. Die empirischen Ergebnisse legen nahe, dass der Wandel in der Korrelation durch die zunehmende Teilzeiterwerbstätigkeit und Bildungsbeteiligung der Frauen, dem Anstieg des Alters bei Geburt sowie dem steigenden Anteil Kinder in Vorschulerziehung bedingt ist.
\end{abstract}

Schlagworte: Fertilität, Frauenerwerbstätigkeit, Korrelation, zusammengefasste Zeitreihen, länder- und zeitspezifische Heterogenität, Determinanten

\begin{abstract}
:
In OECD countries, the cross-country correlation between the total fertility rate and the female labour force participation rate turned from a negative value before the 1980 s to a positive value thereafter. Based on pooled time-series analysis, the literature seems to agree that this change is due to unmeasured country and time heterogeneity with respect to female employment. However, the determinants of this heterogeneity remain unclear. Using data of 16 European countries from 1960-2005, I estimate pooled time-series models of fertility and female labor force participation by applying Prais-Winsten regressions with fixed country and time effects and investigate the changing effect of female labor participation for a set of labor market, educational and demographic variables and indicators of social policy. The empirical findings reveal that the change in the correlation seems to be due to the increasing proportion of females employed part-time, increasing educational attainment of females, increasing age at first birth and increasing gross enrollment ratio of children in pre-primary education.
\end{abstract}

Key words: cross-country correlation, female employment, fertility, pooled time-series, country- and time-specific heterogeneity 


\section{Einleitung}

Während der letzten beiden Dekaden haben sich in OECD-Ländern zwei Entwicklungen vollzogen, die die Aufmerksamkeit von Soziologen, Ökonomen, Demographen sowie die der breiten Öffentlichkeit auf sich gezogen haben. Auf der einen Seite ist in den meisten entwickelten Ländern die Fertilitätsrate unter das Reproduktionsniveau gefallen. begleitet von einem Anstieg der Frauenerwerbsquote. Die Gesamtfertilitätsrate der OECD-Länder ist von einem durchschnittlichen Wert von 2,9 im Jahr 1960 auf einen Wert von 1,6 im Jahr 2005 gefallen, während die Frauenerwerbsquote auf 25,3\% im Jahr 1960 auf 60,4\% im Jahr 2005 stieg.

In Übereinstimmung mit diesen soziodemographischen Entwicklungen wird in der sozialwissenschaftlichen Literatur gemeinhin angenommen, dass sich auf Grund der gegeben gesellschaftlichen Rahmenbedingungen und der schwierigen Vereinbarkeit, Kinder und Erwerbsbeteiligung gegenseitig ausschließen, und sich Frauen deshalb in einer bestimmten Lebensphase entweder für Kinder oder Beruf entscheiden. In der Tat belegen unzählige internationale Studien zum individuellen Arbeitsangebot von Frauen, dass Frauen mit Kindern im Mittel eine geringere Arbeitsmarktbeteiligung aufweisen als kinderlose Frauen (für einen Überblick siehe Schröder/Pforr 2009). Auf der gesamtgesellschaftlichen Ebene sollte dieser Entscheidungsprozess für Kind oder Beruf dann zu einer negativen Beziehung zwischen der Frauenerwerbsquote und der Gesamtfertilitätsrate führen (Becker 1960, 1991; Mincer 1963; Willis 1973; Butz/Ward 1979).

In den letzten Jahren wurde die bis dato als Faktum gesehene negative Beziehung zwischen Fertilität und Frauenerwerbstätigkeit auf der Makroebene in der Fachliteratur wiederholt in Frage gestellt. Verschiedene Autoren zeigen denn auch, dass die jährliche Korrelation zwischen der Frauenerwerbsquote und der Gesamtfertilitätsrate in OECDLändern Mitte der 1980er Jahre einen Wechsel von einem negativen zu einem positiven Wert aufweist (z.B. Ahn/Mira, 2002; Brewster/Rindfuss, 2000; Esping-Andersen, 1999; Pampel, 2001; Rindfuss et al., 2003). Die gemeinhin bekannte negative Beziehung scheint sich somit umgedreht zu haben: Die Länder mit einer niedrigen Fertilität sind nun diejenigen mit einer geringen Frauenerwerbsquote, und die Länder mit einer relativ hohen Fertilität weisen eine hohe Erwerbsbeteiligung der Frauen auf. Abbildung 1 zeigt diese Entwicklung anhand der Daten von 16 europäischen Ländern.

Interessanterweise hat sich nicht nur ein Wandel in der Korrelation zwischen der Fertilität und der Frauenerwerbsquote vollzogen, sondern auch ein Vorzeichenwechsel im Zusammenhang mit anderen sozialen Variablen, etwa dem Erstheiratsalter von Frauen, dem mittleren Alter bei Geburt des ersten Kindes und der Scheidungsrate (Prskawetz et al. 2009; Monnier/de Guibert-Lantoine 1996), dem Anteil außerehelicher Geburten und der Bildungsbeteiligung von Frauen (Sleebos 2003), sowie der wirtschaftlichen Entwicklung gemessen anhand des Human Development Index (Myrskylä et al. 2009). 
Abbildung 1: Korrelation zwischen Fertilität und Frauenerwerbstätigkeit in 16 europäischen Ländern, 1960-2005

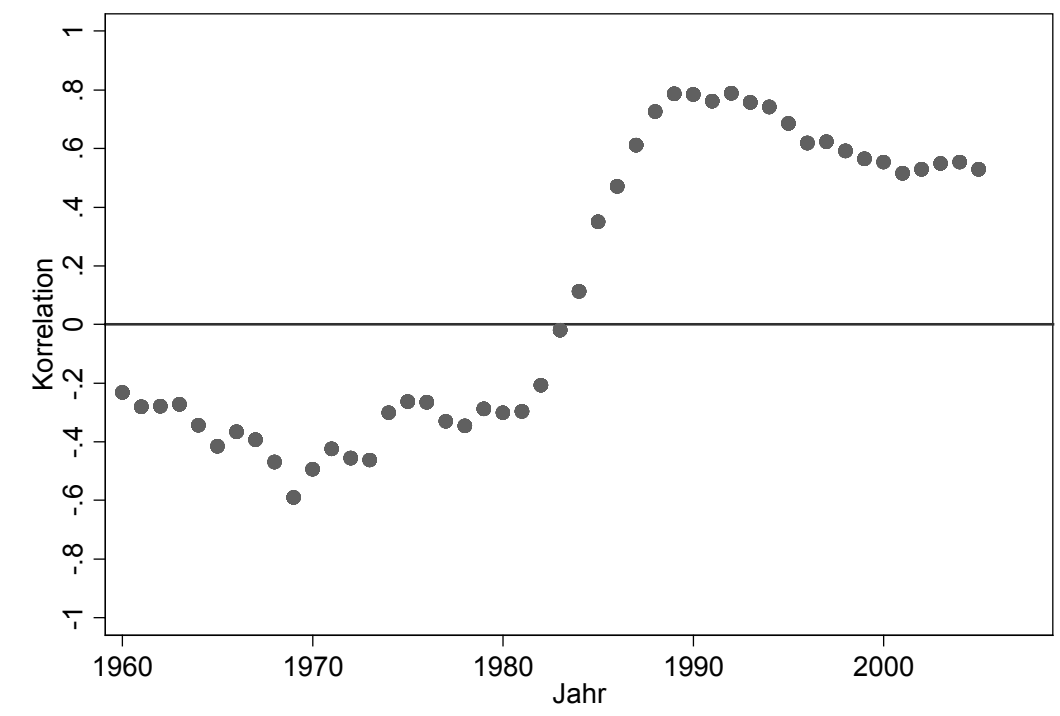

Daten: Siehe Anhang; eigene Berechnungen

Der Vorzeichenwechsel in der Korrelation zwischen der Gesamtfertilitätsrate (TFR) und der Frauenerwerbsquote (FLP) wurde in der Literatur gelegentlich als ein Wechsel in der Beziehung zwischen den beiden Variablen gedeutet (Brewster/Rindfuss, 2000; EspingAndersen, 1999; Rindfuss et al., 2003). Neuere Studien von Kögel (2004) und Engelhardt et al. (2004) zeigen, dass sich weder die Kausalität noch die Zeitreihenbeziehung zwischen den beiden Variablen über die Zeit verändert hat. Kögel (2004) führt die vermeintliche Umkehrung des Zusammengang auf (a) länderspezifische Heterogenität und (b) auf zeit- und länderspezifische Unterschiede in der negativen Beziehung beider Variablen zurück. So hatte z.B. Italien sowohl 1965 als auch 1995 eine geringere Frauenerwerbstätigkeit als Schweden (länderspezifische Heterogenität), wobei der in beiden Ländern zu beobachtende Anstieg der Erwerbsquote in Italien mit einer stärkeren Reduktion der Fertilität ein herging (Unterschiede in der Beziehung der beiden Variablen) (Engelhardt/ Prskawetz 2004; siehe auch Abbildung 2 in Schröder/Pforr 2009). Anhand sechs verschiedener Länder finden Engelhardt et al. (2004) darüber hinaus, dass eine sogenannte Granger-Kausalität in beide Richtungen besteht, was konsistent ist mit simultanen Bewegungen beider Variablen, die durch gemeinsame exogene Faktoren verursacht werden.

Obwohl die genannten Studien ökonometrisch zeigen, dass sich die Zeitreihen beziehung in ihrem Vorzeichen nicht geändert hat, bleiben sie über die genauen Ursachen im Unklaren. Die Studien von Adsera (2004), Ahn/Mira (2002), Castles (2003), Pampel (2001), Engelhardt/Prskawetz (2004) sowie De Laat/Sevilla-Sanz (2007) präsentieren Theorien und Daten, die den mutmaßlichen Wechsel in der Korrelation erklären sollen. Basierend auf familienökonomischen Theorien sowie Überlegungen zur Rolleninkompatibilität werden insbesondere institutionelle Unterschiede sowie Unsicherheiten auf dem 
Arbeitsmarkt als Gründe angeführt, die simultan die Entscheidungen für Familie und Erwerbsbeteiligung beeinflussen. Empirisch wird allerdings in keiner Arbeit explizit der Effekt dieser mutmaßlichen Faktoren auf die zeit- und länderspezifischen Veränderungen in der Beziehung zwischen Fertilität und Frauenerwerbstätigkeit analysiert.

In der vorliegenden Arbeit wird nun meines Wissens erstmals der Effekt der Frauenerwerbstätigkeit auf die Fertilität unter expliziter Berücksichtigung zeit- und länderspezifischer Heterogenität unter Kontrolle diverser Indikatoren aus den Bereichen Arbeitsmarkt, Bildung, Demographie und Sozialpolitik geschätzt, die sowohl die Fertilität als auch die Frauenerwerbstätigkeit beeinflussen. Dabei gestaltet sich der Gang der Analyse wie folgt: Nach einer kurzen Diskussion neuerer theoretischer Ansätze zum Zusammenhang von Fertilität und Frauenerwerbstätigkeit in Abschnitt 2 wird der analytische Rahmen vorgestellt. Sodann werden in Anschnitt 3 die Daten und einige deskriptive Befunde vorgestellt, worauf die graphische Präsentation der Ergebnisse der multivariaten Schätzungen folgt. Abschnitt 4 schließt mit einer kurzen Diskussion und einem Ausblick für künftige Forschung.

\section{Analytischer Rahmen}

In der Ökonomik existieren zwei unterschiedliche Theorien zur Erklärung des Rückgangs der Fertilität und dem Anstieg der Frauenerwerbsbeteiligung: die Neue Haushaltsökonomik und das Modell von Easterlin. Beide Ansätze nehmen für sich in Anspruch, die negative Beziehung dieser beiden Variablen zu erklären; dabei unterscheiden sie sich allerdings in der Identifikation der treibenden Kräfte, wie in den alternativen Bezeichnungen der beiden Ansätze - „,value of time“-Modell und „,relative income“-Modell - zum Ausdruck kommt (Sanderson 1976). Die Neue Haushaltsökonomik (z.B. Wills 1973 und Butz/Ward 1970) fokussiert primär auf Veränderungen im Wert der Zeit der Frauen, während sich die Argumentation von Easterlin $(1980,1987)$ auf Veränderungen im relativen Einkommen durch den demographischen Zyklus stützt.

In der Soziologie und Demographie wird gerne mit der alternativen Hypothese der Rolleninkompatabilität argumentiert. Diese besagt, dass die Inkompatibilität zwischen Familie und Erwerbsbeteiligung durch gesellschaftliche Veränderungen reduziert wurde, wodurch nun in vielen Ländern Familie und Erwerbstätigkeit vereinbar sind. Die drei genannten Theorien sind in der Literatur hinlänglich bekannt und werden u.a. in Engelhardt/Prskawetz (2002) ausführlich diskutiert, so dass ich mich an dieser Stelle kurz fasse (siehe auch Schröder/Pforr 2009). Betont werden soll an dieser Stelle jedoch, dass diese drei Ansätze zwar simultan die Entwicklung von Fertilität und Erwerbsbeteiligung erklären, keiner der Ansätze vermag jedoch explizit die negative Beziehung zwischen Fertilität und Frauenerwerbstätigkeit auf der Mikroebene, die positive Korrelation auf der Makroebene sowie den Wechsel in der Korrelation zu erklären.

Dies leisten De Laat/Sevilla-Sanz (2007) in einem Modell der Haushaltsproduktion, das auf die Heterogenität in Einstellungen bezüglich der Freizeit von Frauen sowie auf die mit der Bereitschaft der Männer sich an der Haushaltsproduktion zu beteiligen verbundenen Externalitäten abstellt. De Laat und Sevilla-Sanz zeigen, dass Haushalte mit weniger egalitären Einstellungen eine ungleichere Verteilung der Aufgaben im Haushalt sowie ei- 
ne geringere Erwerbsbeteiligung der Frauen aufweisen und mehr Kinder haben. Die beiden Autoren nennen diese Beziehung den ,household attitude'-Effekt. Auf der Makroebene zeigen sich Effekte sozialer Externalitäten: Haushalte in weniger egalitären Ländern haben ceteris paribus eine ungleichere Verteilung der Haushaltsaufgaben, eine geringere Frauenerwerbsbeteiligung und auch weniger Kinder. Die Interaktion zwischen der Einstellung zur geschlechtsspezifischen Arbeitsteilung im Haushalt und den Effekten sozialer Externalitäten können nun den Wechsel in der Korrelation verursachen. So zeigen de Laat und Sevilla-Sanz, dass mit dem Anstieg der Frauenlöhne in egalitären Ländern die Beteiligung der Männer an der Haushaltsproduktion steigt, wodurch der Effekt sozialer Externalitäten dominieren kann. Zusammenfassend ist der Beitrag von de Laat und SevillaSanz die erste Arbeit, die eine tatsächliche theoretische Untermauerung für die negative Korrelation auf der Mikroebene, die positive Korrelation auf der Makroebene als auch für den Wechsel in der Korrelation zwischen Fertilität und Frauenerwerbstätigkeit über die Zeit liefert. Ihr zentrales Argument ist dabei, dass Änderungen in sozioökonomischen Trends wie der Anstieg der Frauenerwerbsbeteiligung, dazu führten, dass Unterschiede in Einstellungen, Werten und sozialen Normen ans Tageslicht kamen. Anders gesagt deckten die Veränderungen im sozioökonomischen Kontext bereits bestehende institutionelle und kulturelle Unterschiede zwischen den Ländern auf. Da sich der sozioökonomische Kontext schneller ändern kann als grundlegende kulturelle und soziale Normen, führt dies zu einer Umkehrung der Korrelation zwischen der Fertilität und der Frauenerwerbsbeteiligung.

Eine weitere und recht ähnliche Interpretation in der Umkehrung der Beziehung zwischen Fertilität und zugehörigem Verhalten gibt McDonald (2000). Danach ist die gegenwärtig niedrige Fertilität in modernen Gesellschaften zu interpretieren als ,the outcome of a conflict or inconsistency between high levels of gender equity in individual-oriented social institutions and sustained gender inequity in family-oriented social institutions" (S. 427). McDonald betont die ungleiche Entwicklung hin zu mehr Geschlechtergleichheit in familienorientierten und individualorientierten Institutionen. Der Übergang von hoher zu niedriger Fertilität geht einher mit zunehmenden Rechten für Frauen innerhalb der Familie und im Besonderen der Möglichkeit die Anzahl der Geburten den individuellen Vorstellungen anzupassen. Der außergewöhnliche Anstieg der Geschlechtergleichheit in individual-orientierten Institutionen während des 20. Jahrhunderts, z.B. die Möglichkeit des Erwerbs formaler Bildung und die Partizipation im Arbeitsmarkt, bringt ein Konflikt mit der Rolle als Ehefrau und Mutter aufgrund der fortwährenden geringen Geschlechtergleichheit in der Familie mit sich. „But in a context of persistent relatively low gender equity in family-oriented institutions, high gender equity in individual-oriented institutions results in very low fertility. [...] In a context of high gender equity in individual-oriented institutions, higher gender equity in family-oriented institutions will tend to raise fertility" (McDonald 2000: 438).

Zusammenfassend legen die theoretischen Ansätze nahe, dass die Effekte der traditionellen Fertilitätsdeterminanten wie die Erwerbsbeteiligung von Frauen einhergehen mit dem Einfluss unterschiedlicher sozialer, ökonomischer, institutioneller und kultureller Merkmale. Diese müssen bei der Analyse der Fertilitätsdynamik explizit berücksichtigt werden.

Die vorliegenden empirischen Studien zum Wechsel der Korrelation zwischen Fertilität und Frauenerwerbstätigkeit konzentrieren sich denn auch auf die Ursachen des 
Rückgangs des Fertilität (Adsera 2004; Ahn/Mira 2002; Castles 2003; Pampel 2001; De Laat/Sevilla-Sanz 2003). In einer Erweiterung des theoretischen Modells von Butz/Ward (1979) betonen Ahn/Mira (2002) die Relevanz von Einkommenseffekten, unflexiblen Arbeitszeiten, Arbeitslosigkeit sowie der Kinderbetreuung. Empirische Evidenz präsentieren die beiden Autoren jedoch nur für die aggregierte Arbeitslosigkeit, wodurch ein Großteil der Diskussion auf theoretischen Überlegungen fußt. Basierend auf einem familienökonomischen Ansatz schätzt Adsera (2004) die Effekte von Arbeitsmarktarrangements (geschlechtsspezifische Arbeitslosigkeit und Aktivitätsraten, Dauer des Mutterschutzes und Lohnersatzraten während des Mutterschutzes sowie dem Bruttosozialprodukt) auf die gepoolten Fertilitätsraten von 23 OECD-Ländern. Unter der Annahme eines Rückgangs des Effekts der Frauenerwerbsrate auf die Gesamtfertilitätsrate durch Änderungen in den Präferenzen schätzt Pampel (2001) den Effekt der Erwerbsbeteiligung anhand gepoolter Zeitreihen von 18 Ländern. Castles (2003) beschränkt seine Analysen auf die bivariate Beziehung zwischen der Gesamtfertilitätsrate und verschiedenen Indikatoren der Frauenerwerbstätigkeit, tertiärer Bildungsbeteiligung von Frauen, Anteil der Katholiken und der Scheidungsrate zu zwei Zeitpunkten (1980 und 1998). Die empirische Überprüfung des oben skizzierten theoretischen Modells von De Laat/Sevilla-Sanz (2007) zu Veränderung individueller Einstellungen bezüglich Geschlechterrollen basiert auf international vergleichbaren Querschnittsdaten aus einem einzigen Jahr. Engelhardt/Prskawetz (2004) verwenden ein Set von Arbeitsmarktvariablen, Bildungsindikatoren sowie demographischen und sozialpolitischen Indikatoren zur Erklärung des Vorzeichenwechsels im Korrelationskoeffizienten. Dabei betrachten sie nicht nur die Korrelation der Indikatoren mit der Gesamtfertilitätsrate sondern auch die Zeitreiheninteraktion dieser Variablen mit der Frauenerwerbstätigkeit, beschränken sich jedoch zugleich auf eine rein deskriptive Analyse, in welcher sie die Entwicklung der Indikatoren für Länder mit niedriger, mittlerer und hoher Frauenerwerbsquote nachzeichnen und auf eine Schätzung des Effektes der Erwerbsbeteiligung auf die Fertilität verzichten.

Hier setzt die vorliegende Studie an und liefert einen Beitrag zum Wandel der Fertilität unter Kontrolle der zeit- und länderspezifischen Heterogenität des Effektes der Frauenerwerbsbeteiligung unter Kontrolle von sozialen, ökonomischen und institutionellen Faktoren, die sowohl die Fertilität als auch die Frauenerwerbstätigkeit beeinflussen auf der Makroebene. Zur Analyse des Effektes der Frauenerwerbsquote (FLP) auf die Gesamtfertilität (TFR) werden die länderspezifischen Zeitreihendaten zusammengefasst. Kausalanalyse mit zusammengefassten Zeitreihen ist ungleich komplizierter als mit einfachen Zeitreihen (für einen Überblick siehe Engelhardt 2006). Bei der Anwendung von klassischen Panelmodellen ist die kritische Annahme, dass alle Untersuchungseinheiten durch eine identische Regressionsgleichung zu allen Zeitpunkten beschrieben werden können. Zur Erfassung von räumlicher und zeitlicher Heterogenität nehmen wir im Folgenden feste Regionaleffekte $\left(v_{i}\right)$ und feste Zeiteffekte $\left(v_{t}\right)$ an. Darüber hinaus lassen wir eine Variation des Effektes von FLP in der Zeit zu, indem wir die zeitliche Interaktion von FLP explizit modellieren:

$$
\mathrm{TFR}_{\mathrm{it}}=\alpha+\beta_{1} \mathrm{FLP}_{\mathrm{it}}+\beta_{2} \mathrm{FLP}_{\mathrm{it}} \times \mathrm{t}+v_{\mathrm{i}}+v_{\mathrm{t}}+\varepsilon_{\mathrm{it}}, \mathrm{i}=1, \ldots, \mathrm{N} ; \mathrm{t}=1, \ldots, \mathrm{T} .
$$

$\mathrm{Da}$ in unserem Modell eine starke Autokorrelation bedingt durch zeitlich und räumlich korrelierte Störterme $\left(\varepsilon_{\mathrm{it}}\right)$ vorliegt, was bei Nichtbeachtung zu ineffizienten OLS-Schätzer 
der Koeffizienten führen würde, müssen wir diesem Umstand statistisch Rechnung tragen. Bei zusammengefassten Zeitreihen ist die sogenannte Prais-Winsten-Methode das dominierende Verfahren zur Parameterschätzung bei Vorliegen von serieller Autokorrelation, dessen Ausgangspunkt ein AR(1)-Panel-Modell bildet (Prais/Winsten, 1954). Für die methodischen Details des Schätzverfahrens sei auf Engelhardt/Prskawetz (2005) verwiesen.

Zusammenfassend schätzen wir in den empirischen Analysen ein Basismodell mit fixen Ländereffekten zur Berücksichtigung unbeobachteter länderspezifischer Heterogenität und mit fixen Zeiteffekten zur Erfassung zeitlicher Heterogenität im Niveau der Fertilität. Zur Erfassung der zeitspezifischen Heterogenität in der Beziehung zwischen Fertilität und Frauenerwerbstätigkeit beinhalten alle Modelle zusätzlich Interaktionseffekte zwischen Frauenerwerbstätigkeit und der Zeit. Dieses Basismodell wird in nachfolgenden Schritten erweitert um Indikatoren des Arbeitsmarktes und der Bildungsbeteiligung von Frauen sowie demographische und sozialpolitische Indikatoren, wobei explizit die Interaktion zwischen diesen Indikatoren $(\mathrm{Z})$ und der Frauenerwerbsbeteiligung modelliert wird:

$$
\mathrm{TFR}_{\mathrm{it}}=\alpha+\beta_{1} \mathrm{FLP}_{\mathrm{it}}+\beta_{2} \mathrm{FLP}_{\mathrm{it}} \times \mathrm{t}+\beta_{3} \mathrm{FLP}_{\mathrm{it}} \times \mathrm{Z}_{\mathrm{it}}+\beta_{4} \mathrm{Z}_{\mathrm{it}}+v_{\mathrm{i}}+v_{\mathrm{t}}+\varepsilon_{\mathrm{it}} .
$$

Die Veränderung des zeitabhängigen Effektes der Frauenerwerbstätigkeit unter Kontrolle dieser Faktoren gibt Aufschluss über den Effekt dieser Indikatoren auf die Beziehung zwischen der Fertilität und der Frauenerwerbstätigkeit.

\section{Empirische Analyse}

\section{Daten}

Zur Analyse der Beziehung zwischen Fertilität und Frauenerwerbstätigkeit greifen wir auf jährliche Zeitreihen dieser beiden Variablen aus den Jahren 1960 bis 2005 für 16 europäische Länder zurück. Berücksichtigt wurden je vier Länder aus Nordeuropa (Dänemark, Finnland, Norwegen, Schweden), Westeuropa (Belgien, Frankreich, Großbritannien, Niederlande), Südeuropa (Griechenland, Italien, Portugal, Spanien) und Zentraleuropa (Luxemburg, Deutschland, Österreich, Schweiz). Die Auswahl der Länder basiert u.a. auf der Verfügbarkeit von Daten für unsere Kontrollvariablen (siehe hierzu weiter unten).

Abbildung 2 zeigt anhand der konkreten länderspezifischen Werte für ausgewählte Jahre, wie sich der Wandel in der Fertilität und Frauenerwerbstätigkeit über die Zeit vollzogen hat: Während in den 16 europäischen Ländern Anfang der 1960er Jahre die Fertilitätsrate bei einer insgesamt starken Streuung zwischen 2.2 und 3.15 noch Werte zumeist deutlich über dem Bestandserhaltungsniveau aufweist, sank diese - begleitet von einem Rückgang der Streuung - in den 1970er und 1980er Jahren kontinuierlich ab bei einem gleichzeitigen Anstieg der Frauenerwerbsquote. Mitte der 1980er Jahre zeigt sich dann in nahezu allen Ländern eine Gesamtfertilität von unter 2 mit einer recht geringen Streuung, wobei die Frauenerwerbsrate sich länderspezifisch zwischen 30 und $80 \%$ bewegt. Anfang der 1990er Jahre ist dann in einigen Ländern ein Anstieg in der Gesamtfertilitätsrate zu beobachten, welcher von einem Rückgang der Streuung in der Frauenerwerbsrate bis zur Jahrtausendwende begleitet wird. 
Abbildung 2: Fertilität und Frauenerwerbstätigkeit in ausgewählten Jahren zwischen 1960 und 2005 in 16 europäischen Ländern; geschätzter linearer Zusammenhang und 95\%-Konfidenzintervalle

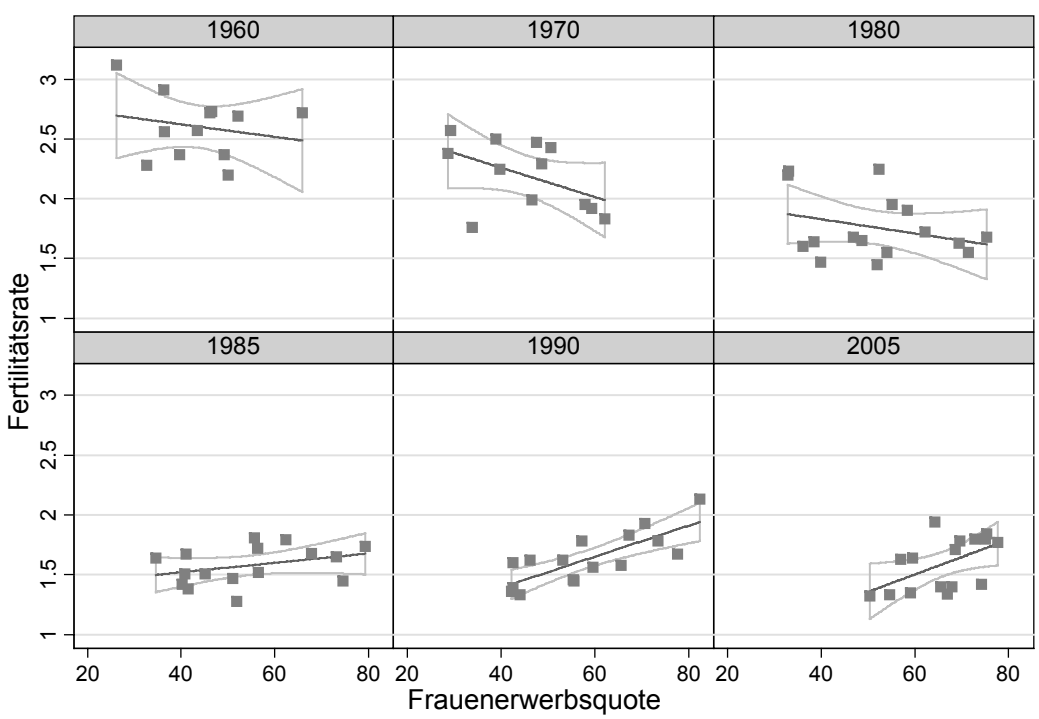

Daten: Siehe Anhang

Abbildung 3: Entwicklung von Fertilität und Frauenerwerbstätigkeit von 1960 bis 2005 in 16 europäischen Ländern

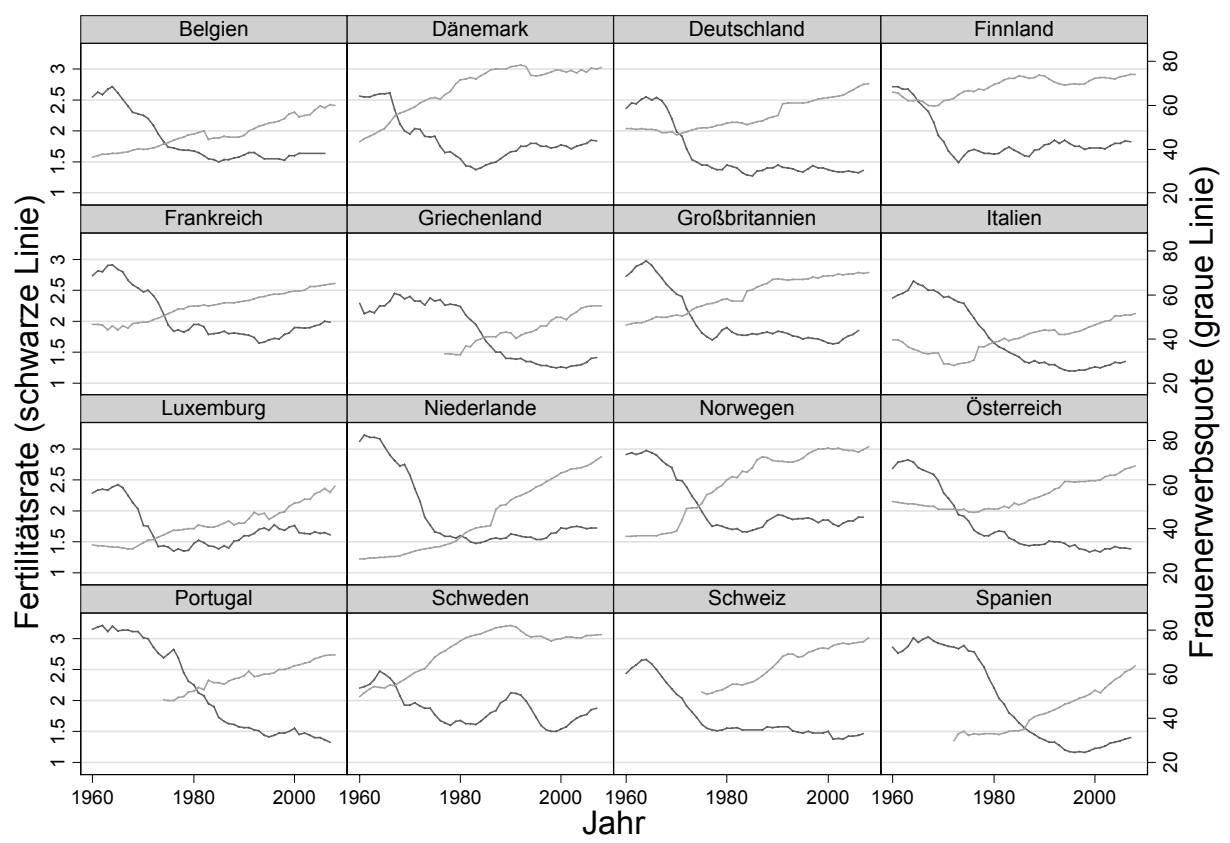

Daten: Siehe Anhang 
Detaillierte Zeitreihen für die einzelnen Länder zeigen denn auch, dass in allen betrachteten Ländern ein deutlicher Anstieg der FLP bei einem gleichzeitigen Rückgang der TFR zu verzeichnen war, wobei der Rückgang der TFR in den meisten Ländern seit Anfang oder Mitte der 1980er Jahre stagniert (Abbildung 3).

Abbildung 4 stellt den Zusammenhang zwischen TFR und FLP im zeitlichen Verlauf auf länderspezifischer Ebene bivariat dar. Es zeigt sich deutlich, dass in allen Ländern ein negativer Zusammenhang zwischen den beiden Variablen zu verzeichnen war, der sich ab einem bestimmten Niveau der Frauenerwerbstätigkeit in einen „Nichtzusammenhang“ gewandelt hat. Zwar steigt die Frauenerwerbstätigkeit in allen Ländern weiter an, dies aber bei einer mehr oder weniger konstanten Fertilität.

Abbildung 4: Zusammenhang von Fertilität und Frauenerwerbstätigkeit von 1960 bis 2005 in 20 OECD Ländern

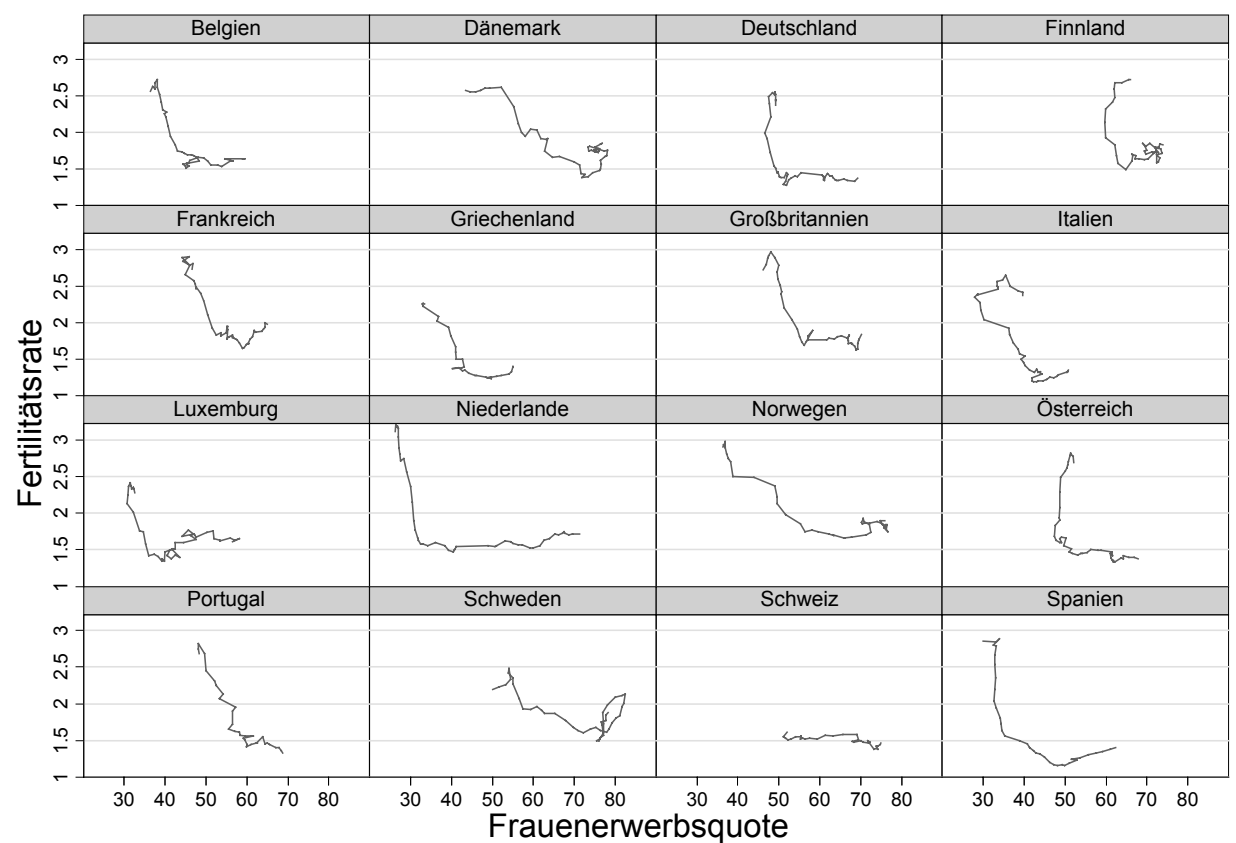

Daten: Siehe Anhang

Als Kontrollvariablen in den multivariaten Analysen dienen diverse Indikatoren aus dem Arbeitsmarkt, Bildungsindikatoren sowie demographische und sozialpolitische Indikatoren, die in der Literatur als Fertilitätsdeterminanten wohlbekannt sind. Als Arbeitsmarktindikatoren werden die Erwerbsrate der Männer, die Arbeitslosenraten für Frauen und Männer und der Anteil Frauen in Teilzeitarbeit an allen erwerbstätigen Frauen sowie die geschlechtsspezifischen Stundenlöhne im verarbeitenden Gewerbe berücksichtigt. Als Bildungsinkatoren wird der Anteil Frauen in tertiärer Ausbildung sowie die durchschnittlichen Schuljahre von Frauen über 25 Jahren herangezogen. Zu den demographischen Indikatoren zählen die Erstheiratsrate und die Scheidungsrate sowie das mittlere Alter bei 
Geburt und bei erster Geburt. Als sozialpolitische Indikatoren wird der Anteil Kinder in Vorschulerziehung, die Höhe der Familienbeihilfe für das erste Kind, die Dauer des Mutterschafts- bzw. Elternurlaubs in Wochen sowie der prozentuale Anteil des Mutterschaftsgeldes am Lohn in den empirischen Analysen berücksichtigt. Die exakten Definitionen der jeweiligen Variablen und die Datenquellen finden sich im Anhang. Leider liegen nicht für alle Indikatoren komplette Zeitreihen für den gesamten Untersuchungszeitraum vor. Vereinzelt fehlende Daten inmitten der Zeitreihen wurden linear interpoliert; fehlende Werte am Anfang oder Ende einer Zeitreihe wurden in den Analysen ausgeschlossen. Da für die meisten Länder die entsprechenden Angaben erst ab 1970 vorliegen, beschränken wir die folgenden multivariaten Analysen auf den Zeitraum 1970 bis 2005. Abbildung 5 gibt einen Überblick über die Entwicklung dieser Variablen.

\section{Multivariate Analysen zusammengefassten Zeitreihen}

Zur Beurteilung der Wirkung unserer diversen sozialen Indikatoren auf den Effekt der Frauenerwerbsbeteiligung für die Gesamtfertilitätsrate wird in einem ersten Schritt das Basismodell geschätzt, das uns im Folgenden als Referenzmodell dient. Dieses Modell beinhaltet sowohl zeit- und länderspezifische fixe Effekte als auch Interaktionseffekte der Frauenerwerbsbeteiligung mit der Zeit. Die exakten Schätzergebnisse sind aufgrund der Vielzahl der geschätzten Parameter hier nicht weiter ausgewiesen. Zur Veranschaulichung der Effekte der Frauenerwerbstätigkeit werden die Ergebnisse im Folgenden graphisch präsentiert. Abbildung 5 zeigt den in der Zeit variierenden Effekt von FLP auf die TFR mit Dummy-Trends basierend auf den Schätzungen des Prais-Winsten-Modells. Wie schon bei der bivariaten Korrelationsanalyse (vgl. Abbildung 1) legen die Schätzergebnisse des Basismodells ein Wechsel im Vorzeichen der Beziehung zwischen TFR und FLP nahe. Seit Mitte der 1980er Jahre ergeben sich für die berücksichtigten Länder mehr oder weniger konstante positive Effekte der FLP auf die Gesamtfertilität.

Wie verändert sich nun der Effekt der FLP auf die TFR unter Berücksichtigung ausgewählter Arbeitsmarktindikatoren? Die der graphischen Darstellung in Abbildung 6 zugrunde liegenden Modelle erweitern das Basismodell um je einen Arbeitsmarktindikator (z.B. die Männererwerbsquote) und berücksichtigen auch die Interaktion dieses Indikators mit der FLP (z.B. Männererwerbsquote*FLP). In der Abbildung sind wiederum die Nettoeffekte von FLP abgetragen, d.h. der Haupteffekt von FLP $\left(\beta_{1}\right)$ korrigiert um die Interaktionseffekte von FLP mit der Zeit $\left(\beta_{2}\right)$ und dem Interaktionseffekt von FLP mit dem Arbeitsmarktindikator $\left(\beta_{3}\right)$. 
Abbildung 5: Entwicklung der Indikatoren in den 16 OECD Ländern ab 1970
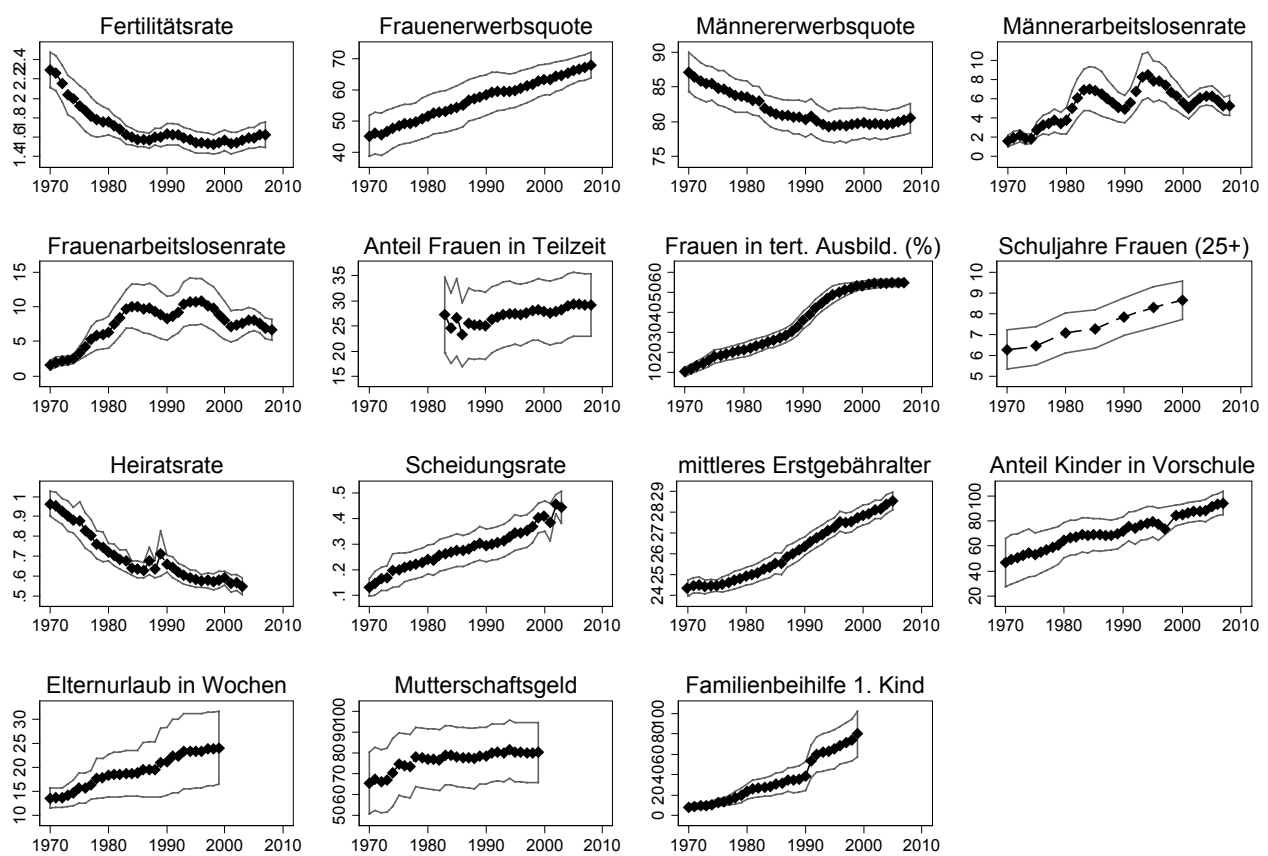

Daten: Siehe Anhang

Abbildung 6: Effekt von Frauenerwerbstätigkeit auf Fertilität unter Kontrolle ausgewählter Arbeitsmarktindikatoren

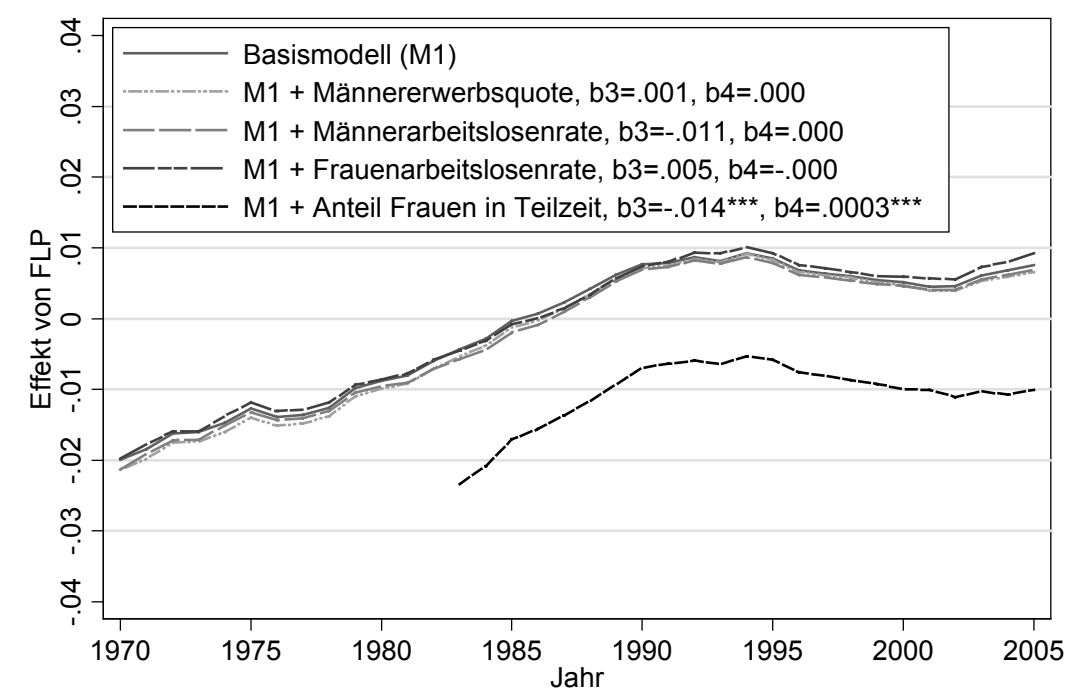

Anmerkung: *** $\mathrm{p}<.001$

Daten: Siehe Anhang; eigene Berechnungen 
Die empirische Untersuchung startet mit einer Analyse der Veränderung der ökonomischen Position von Männern. Indikatoren der ökonomischen Position von Männern beinhalten die Erwerbsrate und die Arbeitslosenrate. Während die FLP über die Zeit in allen Ländern gestiegen ist, war die Männererwerbsrate primär bedingt durch den Rückgang der Partizipation älterer Personen rückläufig (Gruber/Wise 1999). Zusätzlich ist die Arbeitslosenrate der Männer während unseres Untersuchungszeitraumes kontinuierlich gestiegen (Abbildung 5). Diese Entwicklungen zeigen, dass sich die ökonomische Position der Männer verschlechtert hat. Der Familienökonomik zufolge kann diese Entwicklung den Rückgang der Fertilität erklären. In unserem Modell zeigen allerdings weder die Männererwerbsquote noch die Arbeitslosenrate von Männern einen signifikanten Effekt auf die Fertilität. Damit bleibt auch der Effekt von FLP auf TFR unter Berücksichtigung der Interaktion dieser Variablen weitgehend konstant und der Wechsel im Vorzeichen des Zusammenhangs zwischen FLP und TFR erhalten.

Bezüglich der Arbeitslosenrate für Frauen, die wie die der Männer im Untersuchungszeitraum in den meisten Ländern gestiegen ist, zeigt sich ebenfalls kein signifikant negativer Effekt im Modell. Auch für den Effekt der FLP auf die TFR spielt die Arbeitslosenrate der Frauen wie bereits die der Männer offensichtlich keine Rolle. Wie in Abbildung 6 zu sehen ist, verlaufen die Effekte von FLP mit und ohne Berücksichtigung der Arbeitslosenrate von Frauen weitestgehend übereinstimmend. Die Umkehr des Effektes kann somit auch nicht auf die spezifische Definition der FLP zurückgeführt werden, welche auch Erwerbslose mit berücksichtigt, da unter expliziter Berücksichtigung der Arbeitslosigkeit der Effekt von FLP erhalten bleibt.

Entgegen den Vorhersagen der Familienökonomik und der Hypothese der Rolleninkompatabilität hat der Anteil Frauen in Teilzeitarbeit an allen erwerbstätigen Frauen einen signifikant negativen Effekt auf die Fertilität, d.h. je höher die Teilzeiterwerbsquote desto geringer ist die Fertilität. Interessanterweise ist der Interaktionseffekt des Anteils Frauen in Teilzeit mit der FLP signifikant positiv: je höher der Anteil Frauen in Teilzeit, desto mehr steigt somit die TFR mit zunehmender FLP. Unter Kontrolle der Teilzeiterwerbstätigkeit, die sich in den meisten Ländern parallel zur FLP entwickelt, reduziert sich denn auch der Effekt der Frauenerwerbstätigkeit auf die TFR deutlich, der nun im gesamten Untersuchungszeitraum negativ bleibt. Der in den meisten Ländern zu beobachtende Anstieg des Anteils Frauen in Teilzeit hat somit mutmaßlich mit zum Wechsel der Korrelation zwischen TFR und FLP beigetragen.

Eine weitere Erklärung für den Rückgang der Fertilität liegt nach der Familienökonomik in der Zunahme der Bildungsbeteiligung von Frauen, wodurch die Opportunitätskosten steigen und die Fertilität sinken sollte. Sowohl für die Dauer der Schulausbildung der über 25-Jährigen als auch für den Anteil Frauen in tertiärer Ausbildung zeigt sich entsprechend den theoretischen Erwartungen ein signifikant negativer Effekt auf die Gesamtfertilitätsrate, d.h. je länger die Schulbildung dauert, desto geringer ist die Fertilität (Abbildung 7). Unter Berücksichtigung der Bildungsbeteiligung, die mit der Frauenerwerbsrate stark positiv korreliert ist, reduziert dann auch der Effekt der Frauenerwerbsrate auf die Fertilität und bleibt nicht nur im gesamten Untersuchungszeitraum im negativen Bereich sondern verstärkt sich nach einem Rückgang Mitte der 1990er Jahre. Somit trägt auch die zunehmende Bildungsbeteiligung von Frauen zu einem Wechsel in der Korrelation zwischen TFR und FLP bei. 
Abbildung 7: Effekt von Frauenerwerbstätigkeit auf Fertilität unter Kontrolle ausgewählter Bildungsindikatoren

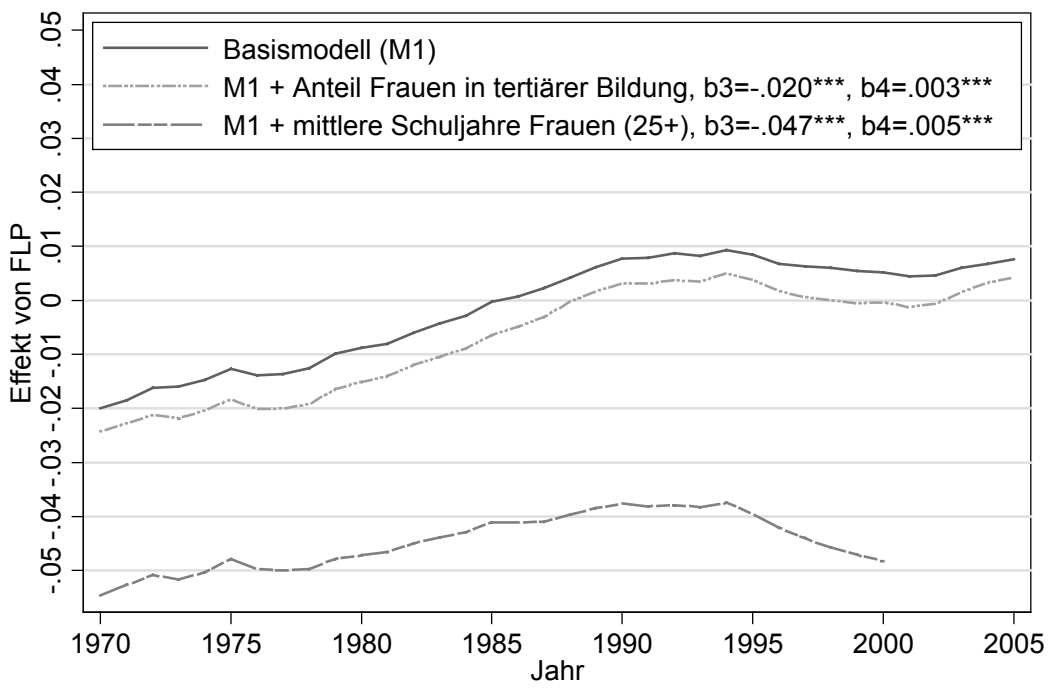

Anmerkung: *** $\mathrm{p}<.001$

Daten: Siehe Anhang; eigene Berechnungen

Im Folgenden betrachten wir den Effekt einiger proximaler Determinanten der Fertilität, die zugleich auch mit der Erwerbsbeteiligung von Frauen interagieren dürften. In allen betrachteten Ländern ist die Erstheiratsrate gesunken, welche mit der Erwerbsbeteiligung negativ korreliert ist. Wie Billari/Kohler (2002) sowie Mamolo et al. (2008) zeigen, kam es auch bezüglich der Korrelation zwischen der Erstheiratsrate und der TFR in unserem Untersuchungszeitraum zu einem Wechsel von einem negativen zu einem positiven Vorzeichen. In Ländern, in denen eine langfristige Paarbindung noch immer die Norm darstellt (z.B. in Italien), sollte jedoch der Rückgang in der Erstheiratsrate noch immer mit einem Rückgang in der Fertilität verbunden sein. In der Schätzung finden sich denn auch ein signifikant positiver Effekt der Erstheiratsrate auf die TFR und ein signifikant negativer Interaktionseffekt mit der FLP (Abbildung 8). Die zeitabhängigen Effekte von FLP auf die TFR verändern sich unter Berücksichtigung der Erstheiratsrate kaum und verlaufen weitestgehend übereinstimmend mit den für das Basismodell geschätzten Effekten. Der Rückgang der Erstheiratsrate ist somit kein Faktor, der zur Umkehr der Korrelation zwischen FLP und TFR beigetragen haben dürfte.

Eine weitere proximale Determinante der Fertilität ist die Scheidungsrate, für die sich ein signifikant negativer Effekt auf die Fertilität im Modell ergibt, d.h. je höher die Scheidungsrate, desto geringer ist die Fertilität. Mit der FLP ist die Scheidungsrate stark positiv korreliert. Unter Berücksichtigung der Interaktion zwischen der FLP und der Scheidungsrate zeigt sich nun für den gesamten Zeitraum ein positiver Effekt von FLP auf die TFR. Interpretiert man zunehmende Scheidungsraten als ein Indikator für den Anstieg der Anzahl egalitärer Partnerschaften und zunehmender finanzieller Unabhängigkeit der Frauen, so passt das Ergebnis in das Erklärungsschema von de Laat und Sevilla-Sanz wonach ein 
Wandel in den Einstellungen zur Haushaltsarbeit für den Wechsel in der Korrelation verantwortlich ist.

Neben der Verehelichung und der Eheauflösung gehört das mittlere Alter bei Geburt des ersten Kinds zu den proximalen Determinanten der Fertilität. Das Erstgebäralter ist von besonderem Interesse hinsichtlich der Aufschiebung von Geburten in ein höheres Lebensalter, was in der Literatur als systematisches Muster von „lowest low fertility“ beschrieben wird (Billari/Kohler 2002). In den empirischen Analysen ergibt sich denn auch für das mittlere Alter bei Geburt des ersten Kindes ein signifikant negativer Effekt auf die Gesamtfertilitätsrate. Mit dem Anstieg des Gebäralters kam es somit zu einem Rückgang der Periodenfertilität. Allerdings verstärkt das Gebäralter den negativen Effekt der Frauenerwerbsbeteiligung auf die Fertilität, wodurch er im gesamten Analysezeitraum im negativen Bereich bleibt. Interessant ist, dass das mittlere Alter bei Geburt des ersten Kindes im Vergleich zu mittleren Gebäralter offensichtlich bis Ende der 1980er Jahre einen größeren Einfluss auf den Effekt der FLP ausübt. Seit Anfang der 1990er Jahre sind die Auswirkungen des Alters bei Geburt des ersten Kindes auf den Effekt der TFR weniger deutlich (Abbildung 8).

Abbildung 8: Effekt von Frauenerwerbstätigkeit auf Fertilität unter Kontrolle ausgewählter demographischer Indikatoren

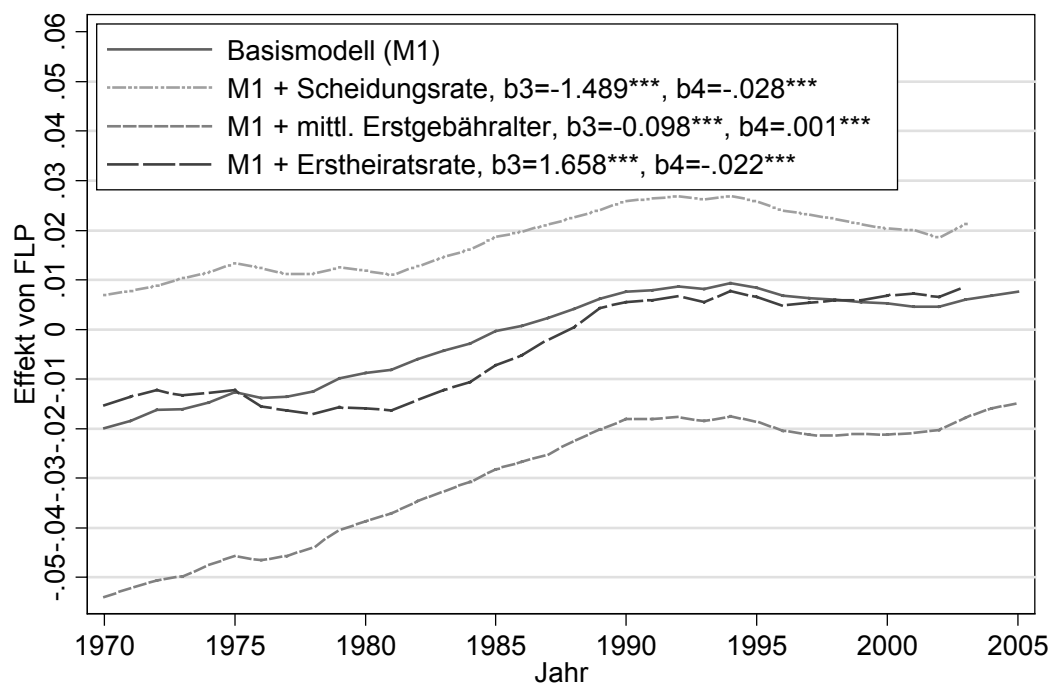

Anmerkung: *** $\mathrm{p}<.001$

Daten: Siehe Anhang; eigene Berechnungen

Betrachten wir schließlich noch die Effekte der sozialpolitischen Indikatoren. Hierzu kalkulieren wir die Höhe der Familienbeihilfe für das erste Kind standardisiert durch die Kaufkraftparität in US-Dollar. Der Effekt der Familienbeihilfe auf die TFR ist in unserem Modell nicht signifikant und der Effekt der FLP auf die TFR bleibt unter Berücksichtigung der Interaktion mit der Familienbeihilfe weitgehend konstant (Abbildung 9). Somit dürfte die Höhe der Familienbeihilfe kein Faktor sein, der zum Wechsel in der Korrelation beigetragen haben dürfte. 
Abbildung 9: Effekt von Frauenerwerbstätigkeit auf Fertilität unter Kontrolle ausgewählter sozialpolitischer Indikatoren

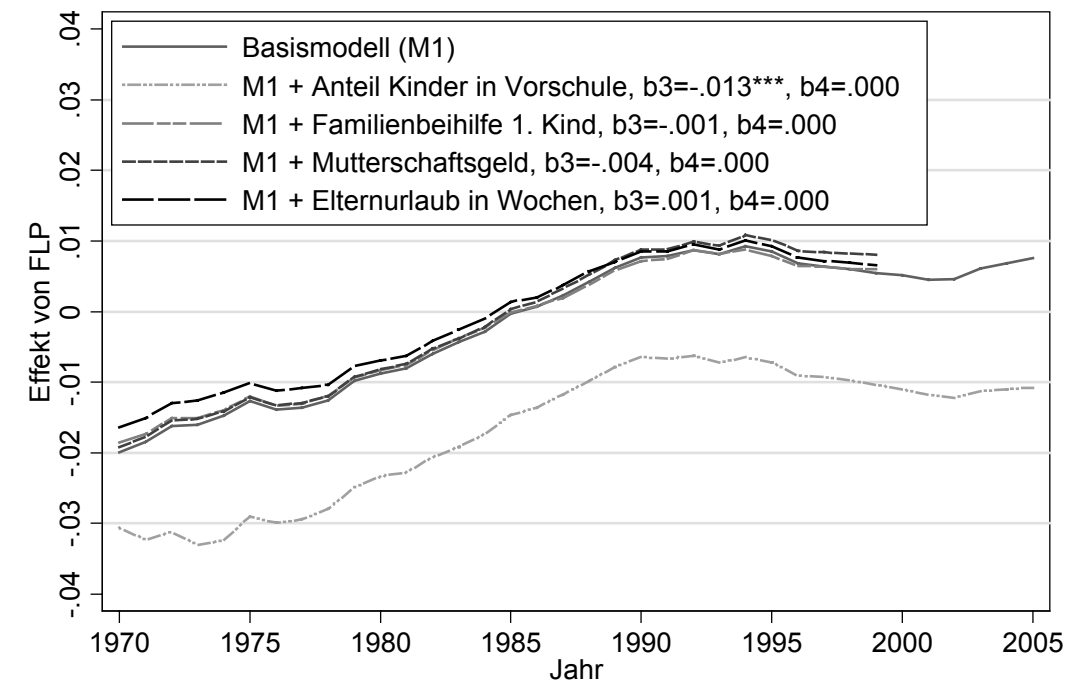

Anmerkung: *** $\mathrm{p}<.001$

Daten: Siehe Anhang; eigene Berechnungen

Das gleiche lässt sich auch für die Höhe des Mutterschaftsgeldes (gemessen am Durchschnittseinkommen von Frauen im verarbeitenden Gewerbe) und die Dauer des Mutterschafts- bzw. Elternurlaubes sagen. Die Effekte auf die TFR sind weder signifikant noch zeigt sich eine wesentliche Änderung des Effektes der FLP auf die TFR.

Anders verhält es sich mit dem Anteil Kinder in Vorschulerziehung, welcher in den meisten Ländern in unserem Untersuchungszeitraum deutlich angestiegen ist (Abbildung 5). Damit hat sich die Vereinbarkeit von Familie und Erwerbstätigkeit deutlich verbessert, was zu einer Erhöhung sowohl der Fertilität als auch der Erwerbstätigkeit beigetragen haben dürfte. Empirisch finden wir allerdings einen signifikant negativen Effekt des Anteils Kinder in Vorschulerziehung auf die Fertilität, welcher die gegenläufige Entwicklung der Zeitreihen wiederspiegelt. Allerdings reduziert sich durch den Anteil Kinder in Vorschulerziehung der Effekt von FLP auf die TFR, der sich von Mitte der 1970er Jahre bis Anfang der 1990er Jahre deutlich verringert hat und seither nur noch leicht negativ ist. Somit dürfte auch der Anstieg der Kinderbetreuung seinen Beitrag dazu geleistet haben, dass sich Mitte der 1980er Jahre die Korrelation zwischen FLP und TFR umgekehrt hat.

\section{Diskussion und Ausblick}

In diesem Artikel wurde analysiert, wie und warum sich die Beziehung der Fertilität und der Frauenerwerbstätigkeit über die Zeit verändert hat. Die empirischen Analysen basieren auf einer Stichprobe von 16 europäischen Ländern, für die Daten zur Fertilität und Frauenerwerbstätigkeit von 1960 bis 2005 vorliegen. Anhand einer Prais-Winston-Regres- 
sion mit AR(1)-Störtermen wird dem speziellen Charakter der zusammengefassten Zeitreihendaten Rechnung getragen. Unter Berücksichtigung von länder- und zeitspezifischer Heterogenität zeigt sich auch in der Panel-Schätzung bis Mitte der 1980er Jahre ein negativer Effekt der Erwerbsbeteiligung von Frauen auf die Fertilität, welcher sich hernach in einen positiven Effekt wandelt.

Zur Erklärung der Umkehr des Effektes greifen wir auf Indikatoren des Arbeitsmarktes, Bildungsindikatoren, sozialpolitische Regelungen sowie proximalen Determinanten der Fertilität zurück und modellieren in einer Erweiterung des Basismodells explizit den Einfluss der Indikatoren auf die Fertilität. Zusätzlich wird berücksichtigt, dass sich der Effekt der Frauenerwerbstätigkeit in Abhängigkeit dieser Faktoren unterscheiden kann.

Die graphische Präsentation der Ergebnisse zeigt deutlich, dass der Wandel in der Korrelation zwischen Fertilität und Frauenerwerbstätigkeit durch die zunehmende Teilzeiterwerbstätigkeit sowie Bildungsbeteiligung von Frauen, dem Anstieg des Alters bei Geburt (insbesondere des ersten Kindes), sowie dem steigenden Anteil Kinder in Vorschulerziehung bedingt ist. Diese Veränderungen gehen vermutlich mit einer Änderung der Einstellungen zur geschlechtsspezifischen Arbeitsteilung im Haushalt einher, welche theoretisch sowohl die negative Beziehung zwischen der Fertilität und der Erwerbsbeteiligung auf der Mikroebene, die positive Beziehung auf der Makroebene als auch den Wechsel in der Korrelation erklären kann. Politische Maßnahmen in Form von direkten monetären Hilfen an Familien mit Kindern scheinen dagegen nicht zu einem Wandel in der Beziehung von FLP und TFR beigetragen zu haben. Auch die von Adsera (2004) aufgrund der visuellen Entwicklung der Zeitreihen präferierte Erklärung, wonach die Zunahme der Arbeitslosigkeit v.a. in südeuropäischen Ländern für den Wechsel des Vorzeichens verantwortlich ist, kann in der vorliegenden Studie nicht bestätigt werden.

Tiefere Einblicke in die zugrundeliegenden Prozesse des Wechsels in der Korrelation ließen sich unter Umständen durch bessere Maße der Erwerbsbeteiligung von Frauen erzielen. So sollten in weiterführenden Analysen insbesondere die Effekte der altersspezifischen Erwerbsbeteiligung auf die Gesamtfertilitätsrate genauer untersucht werden. Darüber hinaus wäre interessant zu wissen, inwiefern die Definition der Erwerbsquote einen Effekt auf die interessierende Korrelation und auf den Effekt der Erwerbsbeteiligung hat. $\mathrm{Da}$ in den Zähler der Erwerbsquote alle Erwerbspersonen, d.h. neben der Zahl der tatsächlich Erwerbstätigen auch die Zahl der Arbeitssuchenden, einfließen, wäre ein bereinigter Indikator aufschlussreich, der nur die tatsächlich erwerbstätigen Frauen wiederspiegelt. Ferner wäre in den Analysen der Effekte der Arbeits-, Bildungs- und sozialpolitischen Indikatoren eine Korrektur der Gesamtfertilitätsrate um sogenannte TempoEffekte, d.h. Verschiebungen der Geburten in ein höheres Lebensalter, aufschlussreich, da bei einer Verschiebung der Geburten in ein höheres Lebensalter die Gesamtfertilitätsrate im zeitlichen Verlauf sinkt. Zur Berücksichtigung der zeitlichen und räumlichen Heterogenität in den Effekten der Makroindikatoren auf die Fertilität, sollte darüber hinaus in weiteren Analysen eine entsprechende Modellierung vorgenommen werden. Zu guter Letzt wäre ein breiteres Spektrum an Makroindikatoren wünschenswert, um weitere Faktoren aufzudecken, die dem Wandel der Korrelation zwischen Erwerbsbeteiligung und Fertilität zugrunde liegen. 


\section{Literatur}

Adsera, A. (2004). Changing fertility rates in developed countries. The impact of labor market institutions. Journal of Population Economics, 17 (1), pp. 17-43.

Ahn, N. \& Mira, P. (2002). A note on the changing relationship between fertility and female employment rates in developed countries. Journal of Population Economics, 15, pp. 667-682.

Barro, R. J. \& Lee, J.-W. (2001). International data on educational attainment: Updates and implications. Oxford Economic Papers, 3, pp. 541-563.

Becker, G. S. (1960). An economic analysis of fertility. Demographic and economic change in developed countries. Princeton, NJ: Princeton University Press.

Becker, G. S. (1991). A treatise on the family. Cambridge, MA: Harvard University Press (enlarged edition).

Billari, F. \& Kohler, H.-P. (2002). Patterns of lowest-low fertility in Europe. Rostock: Max Planck Institute for Demographic Research (MPIDR Working Paper 2002-040).

Brewster, K. L. \& Rindfuss, R. R. (2000). Fertility and women's employment in industrialized nations. Annual Review of Sociology, 26, pp. 271-296.

Butz, W. P. \& Ward, M. P. (1979). The emergence of countercyclical US fertility. American Economic Review, 69, pp. 318-328.

Castles, F. G. (2003). The world turned upside down: Below replacement fertility, changing preferences and family-friendly public policy in 21 OECD countries. Edinburgh: University of Edinburgh (Unpublished manuscript).

de Laat, J. \& Sevilla-Sanz, A. (2007). Working women, men's home time and lowest low fertility. Oxford: University of Oxford, Department of Economics (Economics Series Working Papers 308).

de la Fuente, Á. \& Domènech, R. (2002). Educational attainment in the OECD, 1960-1995. Barcelona: Instituto de Análisis Económico (Unpublished manuscript).

Easterlin, R. A. (1980). Birth and fortune: The impact of numbers on personal welfare. New York: Basic Books.

Easterlin, R. A. (1987). Easterlin hypothesis. In: J. Eatwell, M. Milgate, M. \& P. Newman, (Eds), The new Palgrave: A dictionary to economics 2. New York: The Stockton Press, pp. 1-4.

Engelhardt, H. (2006). Kausalanalysen mit separaten und zusammengefassten Zeitreihen. In: A. Diekmann (Hrsg.), Methoden der Sozialforschung. Wiesbaden: VS Verlag für Sozialwissenschaften, S, 368-395 (Sonderheft 44 der Kölner Zeitschrift für Soziologie und Sozialpsychologie).

Engelhardt, H. \& Prskawetz, A. (2002). On the changing correlation between fertility and female employment over space and time. Rostock: Max Planck Institute for Demographic Research (MPIDR Working Paper WP 2002-052).

Engelhardt, H., Kögel, T. \& Prskawetz, A. (2004). Fertility and female employment reconsidered: A macro-level time series analysis. Population Studies, 58 (1), pp. 109-120.

Engelhardt, H. \& Prskawetz, A. (2004). On the changing correlation between fertility and female employment over space and time. European Journal of Population, 20, pp. 35-62.

Engelhardt, H. \& Prskawetz, A. (2005). A pooled time-series on the relation between fertility and female employment. Vienna: Vienna Institute of Demography of the Austrian Academy of Sciences (European Demographic Research Papers 1),

Esping-Andersen, G. (1999). Social foundations of postindustrial economies. Oxford: Oxford University Press.

Gauthier, A. H. (2002). Comparative family benefits database 1970-2000. (Unpublished database).

Gruber, J. \& Wise, D. A. (1999). Social security and retirement around the world. Chicago \& London: The University of Chicago Press.

Kögel, T. (2004). Did the association between fertility and female employment within OECD countries really change its sign? Journal of Population Economics, 17 (1), pp. 45-65.

McDonald, P. (2000). Gender equality in theories of fertility transition. Population and Development Review, 26, pp. 427-439. 
Mincer, J. (1963). Opportunity costs and income effects. In: C. Christ et al. (Eds), Measurement in economics. Stanford, CA: Stanford University Press.

Myrskylä, M., Kohler, H.-P. \& Billari, F. C. (2008). Advances in development reverse fertility declines. Nature, 460, pp. 741-743,

Pampel, F. C. (2001). The institutional context of population change. Chicago: The University of Chicago Press.

Prais, S. \& Winsten, C. (1954). Trend estimation and serial correlation. Chicago (Cowls Commission Discussion Paper Nr. 383).

Prskawetz, A., Mamolo, M. \& Engelhardt, H. (2009, forthcoming). On the relation between fertility, natality and nuptiality. European Sociological Review.

Rindfuss, R. R., Benjamin Guzzo, K. \& Morgan, S. P. (2003). The changing institutional context of low fertility. Population Research and Policy Review, 22 (5-6), pp. 411-438.

Sanderson, W. C. (1976). On two schools of economics of fertility. Population and Development Review 2, pp. 469-477.

Schröder, J. \& Pforr, K. (2009). Der aktuelle Forschungsstand zum kausalen Zusammenhang zwischen Erwerbstätigkeit und Fertilität bei Frauen. Zeitschrift für Familienforschung/Journal of Family Research, 21, 3/2009. S. 218-244.

Sleebos, J. E. (2003). Low fertility rates in OECD countries: facts and policy responses. Paris: OECD (OECD Social, Employment and Migration Working Papers No. 15).

Willis, R. J. (1973). A new approach to the economic theory of fertility behavior. Journal of Political Economy, 81, pp. S14-S64.

Eingereicht am/Submitted on: 16.02.2009

Angenommen am/Accepted on: 18.06.2009

Anschrift der Autorin/Address of the author:

Prof. Dr. Henriette Engelhardt-Wölfler

Professur für Bevölkerungswissenschaft

Otto-Friedrich-Universität Bamberg

D-96045 Bamberg

E-mail: henriette.engelhardt-woelfler@uni-bamberg.de

\section{Anhang: Variablen, Definitionen und Quellen}

Abhängige Variable:

- $\quad$ Gesamtfertilitätsrate $=$ Die mittlere Anzahl Kinder, die eine Frau im Laufe ihres Lebens gebären würde, wenn ihr Geburtenverhalten dem im Beobachtungszeitraum zu beobachtende Verhalten aller Frauen zwischen 15 und 49 entsprechen würde.

Quellen: Eurostat-Datenbank (http://epp.eurostat.ec.europa.eu).

Arbeitsmarktvariablen:

- $\quad$ Frauenerwerbsquote, Männererwerbsquote = Anteil der erwerbsfähigen (d.h. voll- und teilzeitbeschäftigte sowie aktiv arbeitsuchenden) Frauen bzw. Männer im Alter von 15-64 an der weiblichen bzw. männlichen Bevölkerung in dieser Altersgruppe (in Prozent).

Quelle: OECD Labour Force Statistics (http://stats.oecd.org/wbos). 
- $\quad$ Arbeitslosenquote für Männer und Frauen = Anzahl arbeitsloser Männer/Frauen (in Tausend) bezogen auf die Anzahl erwerbsfähiger Männer/Frauen (in Tausend).

Quelle: Eigene Berechnung basierend auf OECD Labor Force Statistics (http://stats.oecd.org/wbos).

- Anteil der Frauen in Teilzeitarbeit an allen erwerbstätigen Frauen (in Prozent), definiert durch eine Beschäftigung unter 30 Stunden pro Woche. Quelle: OECD Labour Force Statistics (http://stats. oecd.org/wbos).

Bildungsindikatoren:

- Anteil Frauen in tertiärer Ausbildung (female tertiary gross enrolment ratio). Quelle: ab 1999 Unesco, on-line statistics: http://stats.uis.unesco.org.

- Durchschnittliche Schuljahre von Frauen über 25 Jahre.

Quelle: de la Fuente and Domènech (2002), Barro and Lee (2001), verfügbar unter: http://www.cid. harvard.edu/ciddata/ciddata.html.

Demographische Variablen:

- $\quad$ Erstheiratsrate $=$ "The probability of first marriage for a person if he or she were to pass through his/her lifetime conforming to the age-specific first marriage rates of a given year." Quelle: Council of Europe (2005).

- Scheidungsrate $=$ "The probability of divorce for a married person if he or she were to pass through his/her marriage years conforming to the duration-specific divorce rates of a given year." Quelle: Council of Europe (2005).

- Durchschnittsalter der Mutter bei der ersten Geburt. Quelle: Eurostat Datenbank (http://epp.eurostat. ec.europa.eu).

Sozialpolitische Indikatoren:

- Anteil Kinder in Vorschulerziehung an der Bevölkerung im Vorschulalter. Quelle: Comparative Family Benefits Database (Gauthier 2002) und ab 1999 UNESCO Data Centre (http://stats.uis. unesco.org).

- Familienbeihilfe für das erste Kind gemessen in Kaufkraftparität in US-Dollar. Quelle: Comparative Family Benefits Database (Gauthier 2002).

- Elternurlaub in Wochen. Quelle: Comparative Family Benefits Database (Gauthier 2002) .

- Mutterschaftsgeld (prozentual zu dem Durchschnittseinkommen von Frauen im verarbeitenden Gewerbe). Quelle: Comparative Family Benefits Database (Gauthier 2002). 